\title{
Leukemic stem cell phenotype is associated with mutational profile in acute myeloid leukemia
}

\author{
Heejoo Han ${ }^{1, *}$, Ja Min Byun ${ }^{2,}$, Dong-Yeop Shin ${ }^{1,2}$, Sung-Soo Yoon ${ }^{1,2,3}$, Youngil Koh ${ }^{1,2}$, Junshik Hong $^{1,2}$, \\ Inho Kim ${ }^{2}$, Chansup Lee ${ }^{3}$, Hyeonjoo Yoo ${ }^{4}$, Hongseok Yun ${ }^{5}$, Man Jin Kim ${ }^{6}$, Sung Im Cho ${ }^{6}$, Moon-Woo Seong ${ }^{6}$, \\ and Sung Sup Park ${ }^{6}$
}

\begin{abstract}
${ }^{1}$ Center for Medical Innovation, Biomedical Research Institute, Seoul National University Hospital, Seoul; ${ }^{2}$ Division of Hematology and Medical Oncology, Department of Internal Medicine, ${ }^{3}$ Cancer Research Institute, Seoul National University Hospital, Seoul, Korea; ${ }^{4}$ Department of Internal Medicine V, Heidelberg University Hospital, Heidelberg, Germany; ${ }^{5}$ Center for Precision Medicine, ${ }^{6}$ Department of Laboratory Medicine, Seoul National University Hospital, Seoul, Korea
\end{abstract}

Received: January 13, 2020 Revised : April 12, 2020 Accepted: April 26, 2020

\section{Correspondence to}

Dong-Yeop Shin, M.D.

Department of Internal

Medicine, Seoul National

University Hospital, 101

Daehak-ro, Jongno-gu, Seoul

03080, Korea

Tel: $+82-2-2072-2228$

Fax: $+82-2-762-9662$

E-mail: stephano.dyshin@gmail.com https://orcid.org/0000-0003-17538846

*'These authors contributed equally to this work.
Background/Aims: Understanding leukemic stem cell (LSC) is important for acute myeloid leukemia (AML) treatment. However, association of LSC with patient prognosis and genetic information in AML patients is unclear.

Methods: Here we investigated the associations between genetic information and the various LSC phenotypes, namely multipotent progenitor (MPP)-like, lymphoid primed multipotent progenitor (LMPP)-like and granulocyte-macrophage progenitors (GMP)-like LSC in 52 AML patients.

Results: In secondary AML patients, MPP-like LSC was significantly higher than de novo AML $(p=0.0037)$. The proportion of MPP-like LSC was especially high in post-myeloproliferative neoplasm AML $(p=0.0485)$. There was no correlation between age and LSC phenotype. Mutations of KRAS and NRAS were observed in MPP-like LSC dominant patients, TP53 and ASXL1 mutations in LMPP-like LSC dominant patients, and CEBPA, DNMT $3 A$ and IDH1 mutations in GMP-like LSC dominant patients. Furthermore, KRAS mutation was significantly associated with MPP-like LSC expression ( $p=0.0540$ ), and TP53 mutation with LMPP-like LSC expression $(p=0.0276)$. When the patients were separated according to the combined risk including next generation sequencing data, the poorer the prognosis, the higher the LMPP-like LSC expression $(p=0.0052)$. This suggests that the dominant phenotype of LSC is one of the important factors in predicting the prognosis and treatment of AML.

Conclusions: LSC phenotype in AML is closely associated with the recurrent mutations which has prognostic implication. Further research to confirm the meaning of LSC phenotype in the context of genetic aberration is warranted.

Keywords: Acute myeloid leukemia; Leukemic stem cell; Next generation sequencing; Prognosis

\section{INTRODUCTION}

Acute myeloid leukemia (AML) is a clinically heterogeneous disease characterized by multitudes of chromosomal abnormalities and gene mutations. With advances in next generation sequencing (NGS) techniques, more sophisticated risk stratification models have been adapted for better management of the disease [1-3]. Unfortunately resistance to treatment and relapse remains a big challenge, and some feel that the mutation status alone is not enough to predict the prognosis of AML.

Leukemic stem cells (LSCs) are cells with self-renewal 
capacity to repopulate a leukemia and often thought as the major cause of resistance and relapse despite their low frequency [4-6]. The origin of these cells, whether it be from normal hematopoietic stem cells or from more mature progenitors that gain stem-ness features, may differ among patients [7-9], but previous reports on the prognostic implications of LSCs support the importance of eliminating LSCs to improve AML clinical outcomes [4]. As such, understanding the relationship between LSC and genetically defined sub-clones can, in turn, help to understand the heterogeneity of AML [10]. However, to date, there are only a few reports specifically focusing on this topic. To this end, we conducted this study to (1) examine the phenotypic diversity of AML-LSC, (2) explore the association between AML-LSC phenotypes and gene mutations, and (3) investigate the prognostic implications of AML-LSCs.

\section{METHODS}

\section{Study design and subjects}

Newly diagnosed adult AML patients, defined as 18 years or older, were included for this study. Patients with acute promyelocytic leukemia were excluded. The patient selection and bone marrow sample acquisition was done between October 2016 and July 2018. At the end, a total of 52 patients were included for analyses. Their medical records were reviewed and analyzed for demographics, baseline disease characteristics, factors related to treatment, response to treatment, adverse events including treatment related mortality, and survival outcomes. Data available up to March 2019 were used. Their bone marrow samples collected at AML diagnosis were subjected to flow cytometry and NGS.

The diagnosis of AML was made according to the WHO classification of hematopoietic neoplasms [11], which requires identification of $20 \%$ or more leukemic blasts in the bone marrow. Secondary AML was defined as AML following myelodysplastic syndrome (MDS) or myeloproliferative neoplasm (MPN) confirmed prior to the diagnosis of AML. Cytogenetic studies were performed onsite, whose satisfactory performance was monitored by a national external quality assurance scheme. Bone marrow cells were cultured for 24 hours then karyotype was analyzed using the standard G-banding technique.
The karyotypes were constructed and chromosomal abnormalities were reported in accordance with the International System for Human Cytogenetic Nomenclature [12]. Prognostic grouping of cytogenetics was performed according to Southwest Oncology Group (SWOG) criteria [13]. Risk stratification was mainly based on cytogenetics, and NGS results for those with available data, according to 2019 National Comprehensive Cancer Network (NCCN) guidelines [14].

The study was conducted in compliance with all national and international ethical standards for research with humans and for research using radiopharmaceuticals. This study was conducted according to the Declaration of Helsinki and was approved by the Institutional Review Board of Seoul National University Hospital (IRB No. H-1902-140-101). Written informed consent was obtained from all patients. All authors had access to the study data and reviewed and approved this study.

\section{Flow cytometry}

Mononuclear cells were isolated from the patient's bone marrow aspirates by ficoll gradient centrifugation and cryopreserved in serum-free medium. Stored cells were thawed to Iscove's Modified Dulbecco's Medium and washed with fluorescence-activated cell sorting (FACS) buffer (2\% fetal bovine serum, Dulbecco's phosphate-buffered saline). Cells were stained with following anti-human monoclonal antibodies: $\mathrm{CD}_{45} \mathrm{APC} /$ cy7 (557833), CD34-APC (555824), CD38-BV421 (562445), CD9oPE (555596), CD123-PE/Cy7 (560826), and CD45RA-PerCP/ Cy5.5 (563429) (BD Bioscience). Analyses were performed on a FACSCanto II (High Throughput Sampler, BD Bioscience, Franklin Lakes, NJ, USA) and FlowJo version 10.0 program (BD Bioscience). First, the expression profiles of $\mathrm{CD}_{34}$ and $\mathrm{CD}_{3} 8$ markers were analyzed (Fig. 1A). Then, the phenotypes of LSCs were further analyzed per methods proposed by Goardon et al. [15]; multipotent progenitor (MPP)-like LSC $\left(\mathrm{CD}_{34} 4^{+} \mathrm{CD}_{3} 8^{-}\right.$ $\left.\mathrm{CD}_{90}{ }^{-} \mathrm{CD}_{45} \mathrm{RA}^{-}\right)$, lymphoid primed multipotent progenitor (LMPP)-like $\mathrm{LSC}\left(\mathrm{CD}_{34}{ }^{+} \mathrm{CD}_{3} 8^{+} \mathrm{CD}_{90} \mathrm{O}^{-} \mathrm{CD}_{45} \mathrm{RA}^{+}\right)$and granulocyte-macrophage progenitors (GMP)-like LSC $\left(\mathrm{CD}_{34}{ }^{+} \mathrm{CD}_{3} 8^{+} \mathrm{CD}_{123}{ }^{+} \mathrm{CD}_{45} \mathrm{RA}^{+}\right)$. The $\mathrm{CD}_{45}$ and side scatter properties were used for gating bulk leukemic blast cells and sub-gated from the cells [16], as shown in Fig. 1B. End results showed that there was a dominantly expressed LSC phenotype in each patient (Fig. 1C). In some 


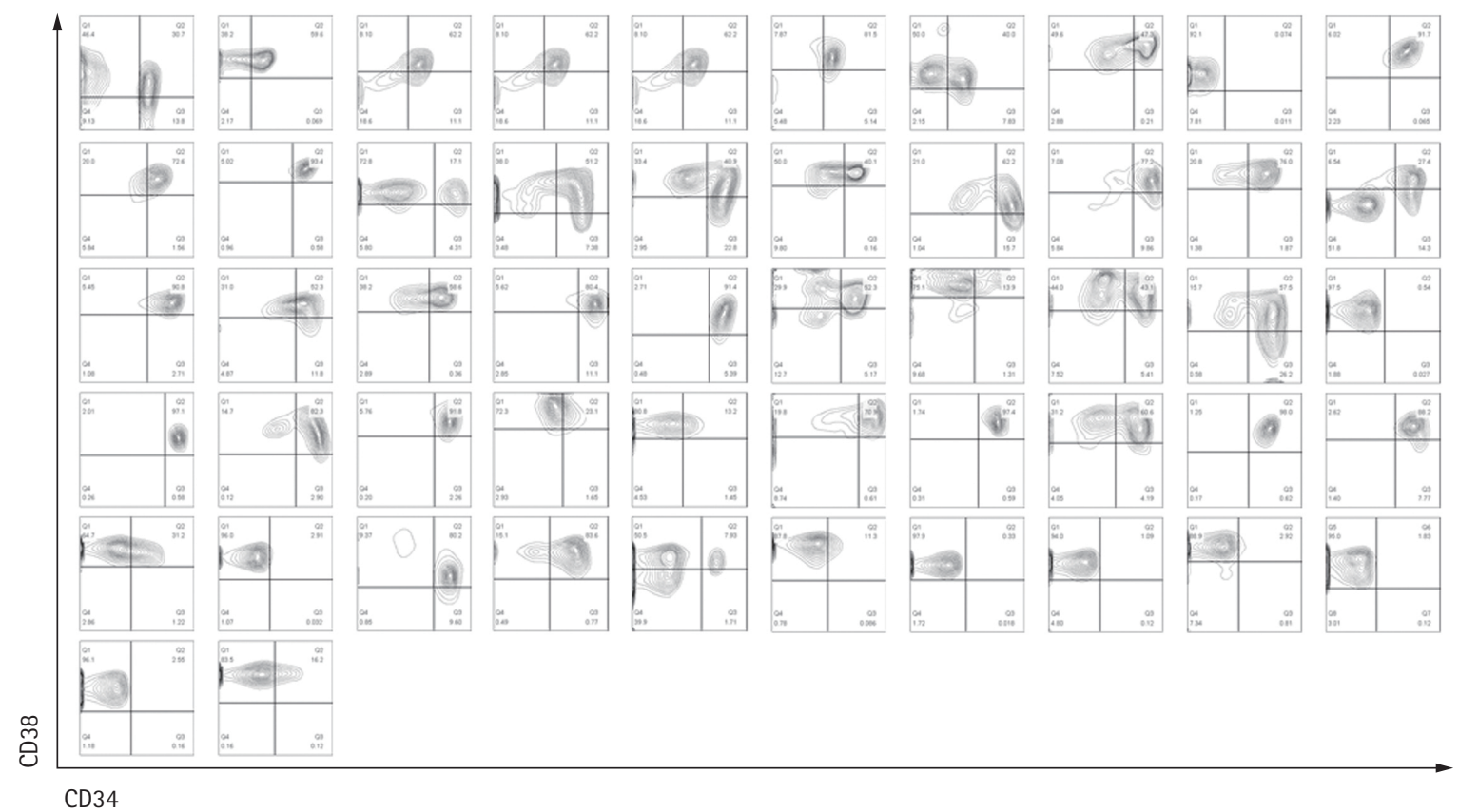

$\mathbf{A}$

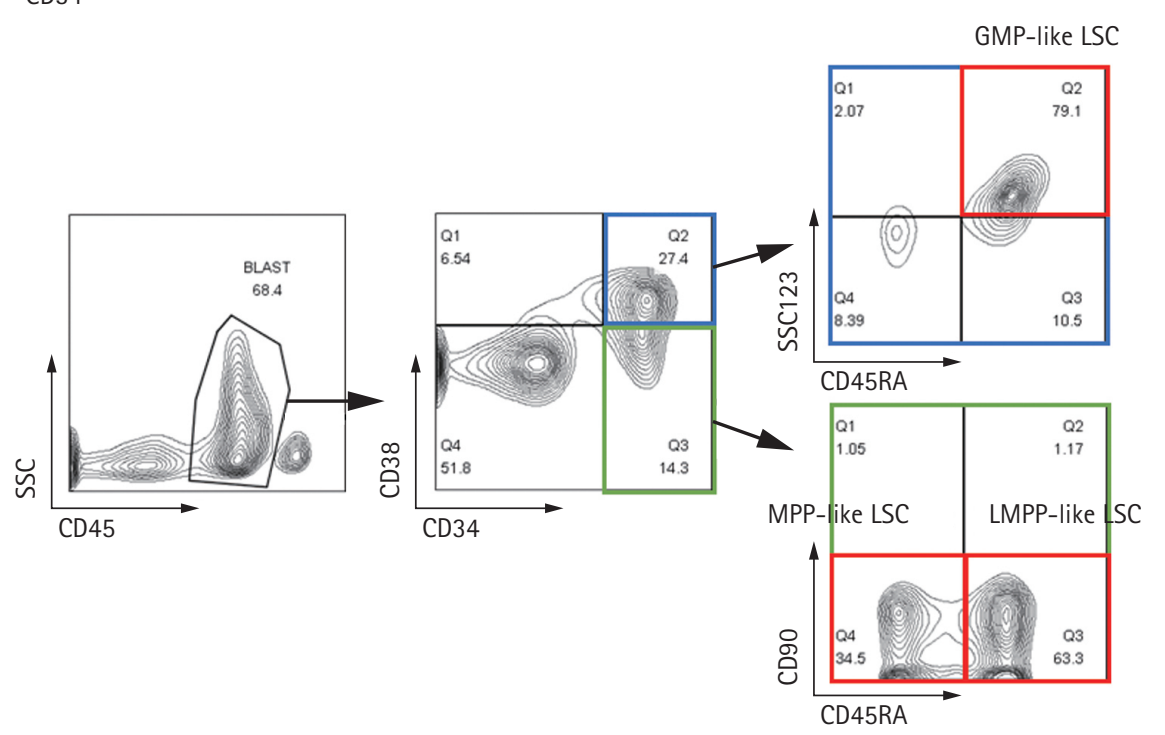

B

LMPP-like LSC dominant

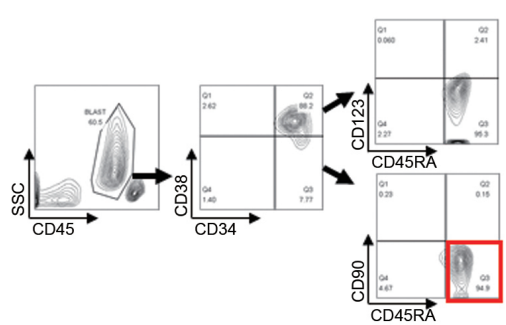

GMP-like LSC dominant

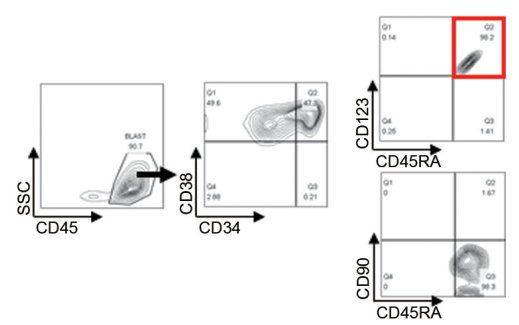

Figure 1. Phenotypic diversity of acute myeloid leukemia (AML) leukemic stem cells (LSCs). (A) CD34 and CD38 surface marker profiles of all 52 patients. (B) Gating strategy for AML-LSCs. (C) Three distinct populations were analyzed as LSC phenotypes: left, multipotent progenitor (MPP)-like LSC; middle, lymphoid primed multipotent progenitor (LMPP)-like LSC; right, granulocyte-macrophage progenitors (GMP)-like LSC. 
cases, two or more LSC phenotypes co-existed. The percentage of cell fractions satisfying each LSC phenotype markers in $\mathrm{CD}_{34}{ }^{+}$cells was calculated.

\section{Targeted capture and massive parallel sequencing}

The DNA capture probes for 76 target genes were designed using the Agilent SureDesign web-based application (https://earray.chem.agilent.com/suredesign/, Agilent Technologies, Santa Clara, CA, USA). The target regions included protein coding exons with $10 \mathrm{bp}$ intron flanking regions and hot spot regions of the 20 genes involved in recurrent translocations. DNA was extracted on a Chemagic 360 instrument (Perkin Elmer, Baesweiler, Germany). The genomic DNA was sheared using Covaris S220 focused-ultrasonicator (Covaris, Woburn, MA, USA). We used 50 ng of total input genomic DNA. A library preparation was performed according to Agilent's SureSelectQXT Target Enrichment protocol (Agilent Technologies). Paired-end 150-bp sequencing was using NextSeq 550 Dx platform (Illumina, San Diego, CA, USA). Targeted sequencing raw data was obtained in FASTQ format.

\section{Secondary analysis using in-house bioinformatics pipeline}

The produced sequencing data was analyzed using the Seoul National University Hospital First Hemic Panel Analysis Pipeline (FHPAP). First, we performed the quality control of the FASTQ file and analyzed only the data that passed the criteria. Pair-end alignment to HG19 reference genome was performed using BWA-men and the GATK Best Practice $[17,18]$. After finishing the alignment step, an "analysis-ready BAM" was produced and a second quality control is performed to determine if further variant calling is appropriate. In the pipeline, SNV, InDel, CNV, translocation, FLT3 ITD, and KMT2A PTD were analyzed using at least more than two analysis tools including in-house and open-source software. Along with in-house software, GATK UnifiedGenotyper, SNVer, LoFreq were used for SNV/InDel detection [1820], Delly and Manta for Translocation discovery [21,22], and THetA2 and CNVKit for purity estimation and CNV calling [23,24], respectively. FLT3 ITD and KMT2A PTD were analyzed by only the in-house software. Detected variants was annotated by SnpEff with various database such as RefSeq, COSMIC, dbSNP, ClinVar, and gno$\mathrm{mAD}$. Then germline variant was filtered using the pop- ulation frequency of these databases $(>1 \%$ population frequency) [25-30]. Finally, the variants were confirmed throughout a comprehensive review of a multidisciplinary molecular tumor board.

\section{Statistical analysis}

The relapse free survival (RFS) and overall survival (OS) curves were estimated using the Kaplan-Meier method. The RFS was derived from the date AML diagnosis to that of relapse or death from any cause, while the OS was defined as the time from AML diagnosis to death from any cause. If patients survived, RFS and OS was censored on the last date of follow-up when no death or relapse was confirmed. Complete remission rate, based on the revised recommendations of the Center for International Blood and Marrow Transplant Research (CIBMTR) group, with induction was compared. Induction TRM was also analyzed, and defined as mortality during induction chemotherapy. The differences between groups were assessed using a Mann-Whitney test or one-way analysis of variance for continuous variables, and Pearson chi-square test for categorical variables, as indicated. All data were analyzed using the SAS Enterprise Guide version 6.1 (SAS Institute, Cary, NC, USA) and the statistical software R (www.r-project.org). A p value of < 0.05 was considered statistically significant.

\section{RESULTS}

\section{Patient characteristics, AML subtypes and LSC phe- notypes}

The baseline characteristics of the enrolled patients are presented in Table 1. There were 40 de novo AML and 12 secondary AML patients. Among secondary AML patients, there were four post-MPN AML patients and eight post-MDS AML patients. The median age of the whole group was 62 years.

When different LSC phenotypes were considered, secondary AML group showed higher fraction of $\mathrm{CD}_{34}{ }^{+} \mathrm{CD}_{3} 8^{-}$cells $(p=0.0245)$ and MPP-like LSCs $(p=$ 0.0037) compared to de novo AML group (Fig. 2A). When secondary AML was further classified into post-MPN versus post-MDS, there was a statistically significant increase in percentage of MPP-like LSCs in post-MPN AML compared to post-MDS AML ( $p=0.0485)$ (Fig. $2 B$ ). 
Table 1. Baseline characteristics

\begin{tabular}{|c|c|c|c|c|}
\hline Characteristic & Total & De novo AML & Secondary AML & $p$ value \\
\hline Number of patients & 52 & 40 & 12 & NA \\
\hline Age, yr & $62(23-88)$ & $63(23-88)$ & $60(40-81)$ & 0.656 \\
\hline$\leq 65$ & $33(63.5)$ & $26(65.0)$ & $7(58.3)$ & \\
\hline$>65$ & $19(36.5)$ & $14(35.0)$ & $5(41.7)$ & \\
\hline Sex, male & $33(63.5)$ & $26(65.0)$ & $7(58.3)$ & 0.674 \\
\hline \multicolumn{5}{|l|}{ Cytogenetic risk } \\
\hline Low & $6(11.5)$ & $6(15.0)$ & $\circ(0.0)$ & 0.001 \\
\hline Intermediate & $38(73.1)$ & $32(80.0)$ & $6(50.0)$ & \\
\hline High & $8(15.4)$ & $2(5.0)$ & $6(50.0)$ & \\
\hline \multicolumn{5}{|l|}{ Combined risk ${ }^{\mathrm{a}}$} \\
\hline Low & $7(13 \cdot 5)$ & $7(17.5)$ & 0 & 0.037 \\
\hline Intermediate & $19(36.5)$ & $15(37 \cdot 5)$ & $4(33 \cdot 3)$ & \\
\hline High & $12(23.1)$ & $6(15 \cdot 0)$ & $6(50.0)$ & \\
\hline Unknown & $14(26.9)$ & $12(30.0)$ & $2(16.7)$ & \\
\hline Extramedullary involve & o & o & 0 & NA \\
\hline \multicolumn{5}{|l|}{ Laboratory findings } \\
\hline Bone marrow blast, \% & $63.7 \pm 22.4$ & $66.5 \pm 20.2$ & $54 \cdot 5 \pm 27.6$ & 0.281 \\
\hline WBC count, /L & $29,169 \pm 54,648$ & $31,740 \pm 61,259$ & $20,599 \pm 20,903$ & 0.334 \\
\hline Platelet count, $\times 10^{9} / \mathrm{L}$ & $86.1 \pm 72.0$ & $81.3 \pm 52.5$ & $102.1 \pm 117.9$ & 0.564 \\
\hline Hemoglobin, g/dL & $9.2 \pm 2.0$ & $9.0 \pm 2.0$ & $9.8 \pm 2.2$ & 0.292 \\
\hline
\end{tabular}

Values are presented as median (range), number (\%), or mean \pm standard deviation.

AML, acute myeloid leukemia; NA, not applicable; WBC, white blood cell.

${ }^{\mathrm{a}}$ Combined risk refers to risk stratification based on available next generation sequencing data including $T P_{53}, A S X_{1}, R_{U N} X_{1}$ mutation status.

Collectively, secondary AML, particularly post-MPN AML, was associated with higher fraction of the MPPlike LSC phenotype compared to its de novo counterpart.

To determine the association between the age and LSC phenotype compositions, patients were divided into two group according to age at AML diagnosis at cutoff of 65 years (Supplementary Fig. 1). The $\mathrm{CD}_{34}{ }^{+} \mathrm{CD}_{3} 8^{-}$cell fraction was higher for younger patients (i.e., $\leq 65$ years), but there were no differences in LSC phenotypes between the two age groups.

\section{Risk stratification, survival and LSC phenotypes}

While data on conventional cytogenetics was available in all patients, the NGS data was not available in 14 patients due to lack of quality-assured samples. The majority of the patients were classified as intermediate risk by both cytogenetics risk stratification and combined risk strat- ification based on NGS data (Table 1). No correlation was seen between cytogenetics risk and LSC phenotypes (Supplementary Table 1). On the other hand, LMPP-like LSC was predominant in the poor risk group by combined risk stratification $(p=0.0052)$ (Fig. 3). This was true for both de novo AML $(\mathrm{n}=28, \mathrm{p}=0.0401)$ and secondary $\operatorname{AML}(\mathrm{n}=10, p=0.0871)$ patients (Supplementary Table 2).

For survival analyses, the patients were grouped according to their dominant LSC phenotypes (Fig. 4). There were ten patients in MPP-like LSC group, 11 in LMPP-like LSC group, and 31 in GMP-like LSC group. For the entire cohort, the mean RFS was 10.8 months and mean OS 12.3 months (Supplementary Table 3). Supplementary Fig. 2 represents the survival curves of the patients undergoing treatment per dominant LSC phenotype. In light of small number of patients with heterogeneous treatment schema, only those who un- 
MPP-like LSC

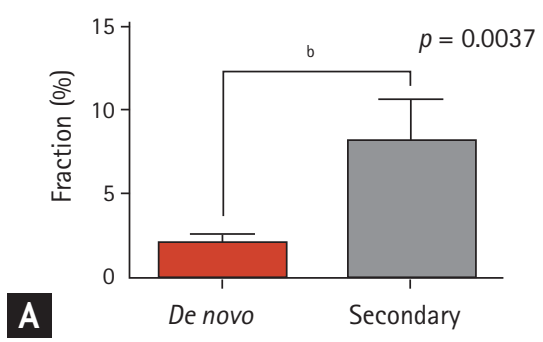

MPP-like LSC

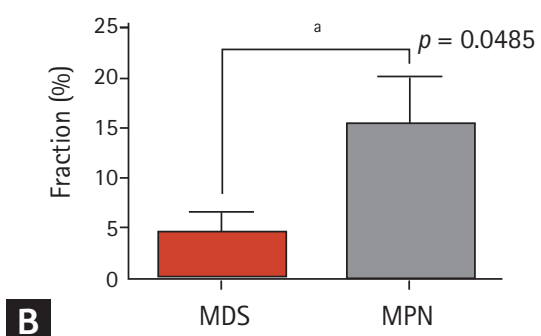

LMPP-like LSC

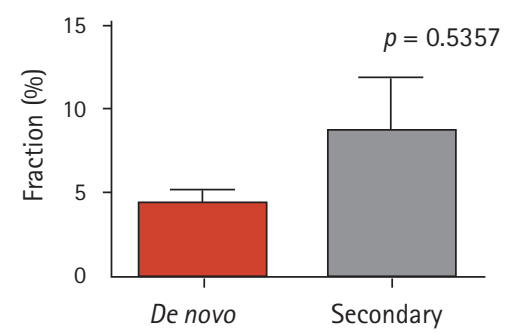

LMPP-like LSC

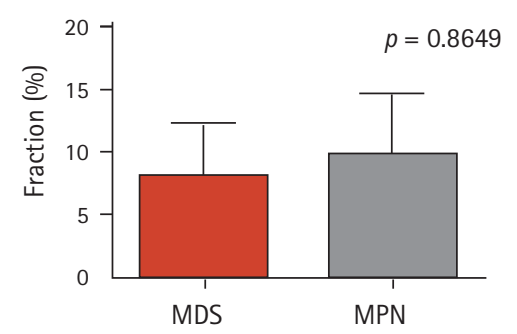

GMP-like LSC

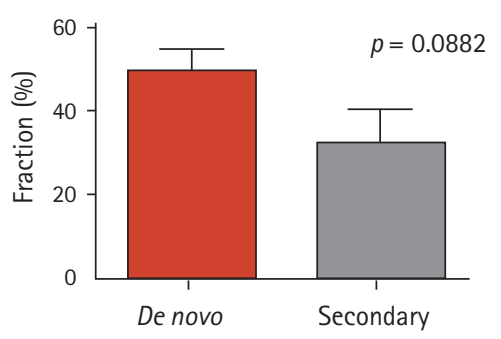

GMP-like LSC

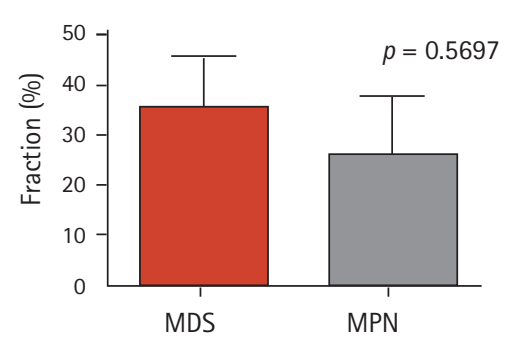

Figure 2. Leukemic stem cells (LSCs) phenotypes and acute myeloid leukemia (AML) subtypes. (A) The multipotent progenitor (MPP)-like LSCs are present in higher fraction in secondary AML patients compared to de novo AML patients. (B) Post-myeloproliferative neoplasm (MPN) AML is associated with higher fraction of MPP-like LSCs compared to post-myelodysplastic syndrome (MDS) AML. The graphs were drawn using a percentage that meets cell surface markers, which means each cell. Error bars indicate mean \pm standard deviation. LMPP, lymphoid primed multipotent progenitor; GMP, granulocyte-macrophage progenitor. ${ }^{\mathrm{a}} \mathrm{p}<0.05,{ }^{\mathrm{b}} \mathrm{p}<0.01$.

derwent treatment regardless of intensity were included for survival analyses. Furthermore, to compensate the innate differences in survival among different AMLs, de novo AML and secondary AML were analyzed separately. There was no correlation between RFS or OS and LSC phenotype in either group.

\section{Individual mutations and LSC phenotypes}

The mutational landscape of enrolled patients is shown in Fig. 4. We looked for specific mutations that were enriched in each LSC phenotype group. Mutations in CEP$B A$ ( 6 out of 7), DNMT $3 A$ (4 out of 7), and IDH1 (3 out of 5 ) were predominantly clustered in GMP-like LSC group. On the other hand, mutations in NRAS (4 out of 6 ) and KRAS (3 out of 4) were dominantly found in MPP-like LSC group, while mutations in TP53 (4 out of 5) and ASXL1 (5 out of 6) were often found in LMPP-like LSC group.

This observation was verified by comparing the LSC phenotype composition of specific mutation carriers versus non-carriers (i.e., wild-type). The differences in LSC expression per CEBPA, DNMT $3 A$, and IDH1 mu- tations were not statistically significant. Interestingly, KRAS mutants showed higher fraction of MPP-like LSC compared to KRAS wild-types (Fig. 5). Also, the fraction of GMP-like LSC was significantly lower in KRAS mutants. NRAS mutants also showed similar tendencies, but the difference did not reach statistical significance. For TP53 and ASXL1 mutants, LMPP-like LSC was present in higher fraction. For TP53 mutants, the expression of LMPP-like LSC was significantly higher by 3.4 -folds $(p=0.0276)$ compared to wild-type. ASXL1 mutants also showed trends towards higher expression of LMPP-like $\operatorname{LSC}(p=0.0654)$.

\section{DISCUSSION}

The importance of our study lies in that we showed for a given AML patients there is a dominant LSC phenotype and LSCs are associated with clinical outcomes, supporting the significance of cancer stem cell model for human AML. First of all, based on detailed characteriza- 
MPP-like LSC
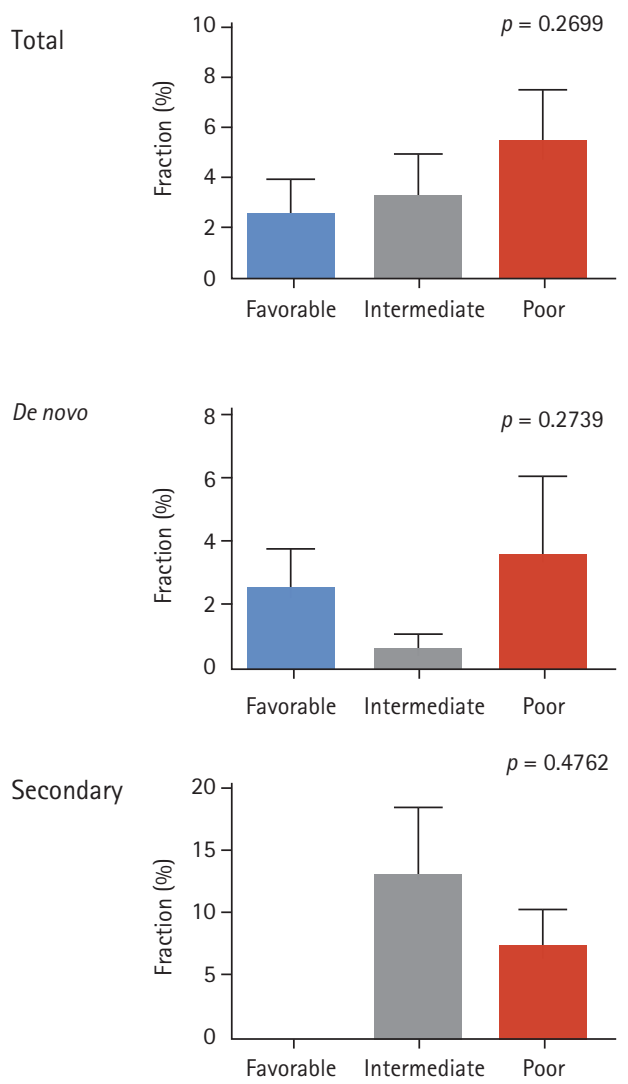

LMPP-like LSC
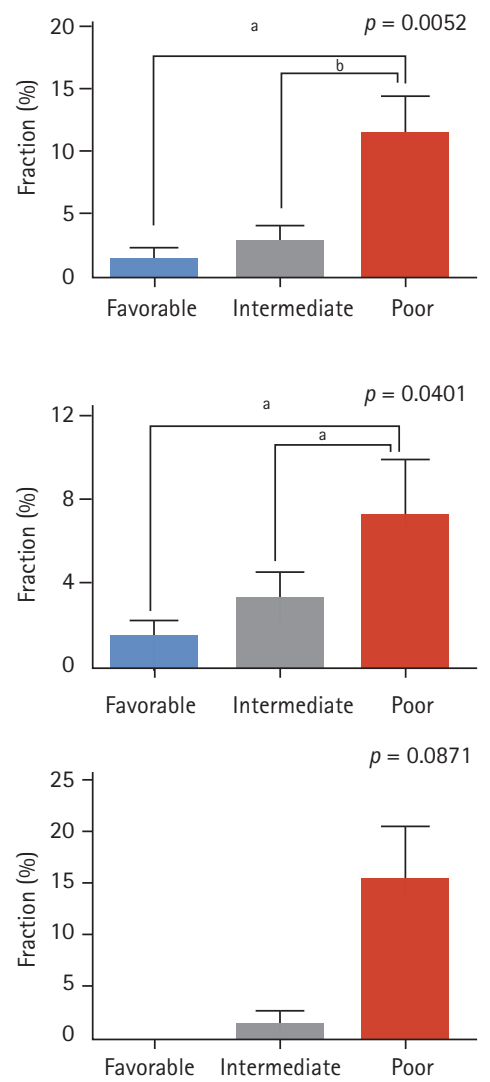

GMP-like LSC
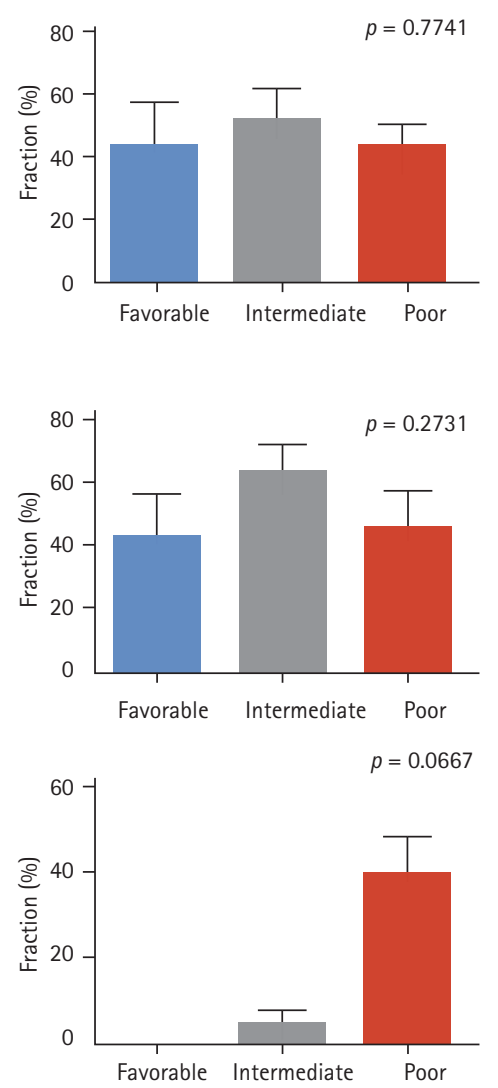

Figure 3. The association between acute myeloid leukemia risk stratification based on next generation sequencing data and leukemic stem cells (LSCs) phenotypes. Error bars indicate mean \pm standard deviations. MPP, multipotent progenitor; LMPP, lymphoid primed multipotent progenitor; GMP, granulocyte-macrophage progenitor. ${ }^{\mathrm{a}} \mathrm{p}<0.05,{ }^{\mathrm{b}} \mathrm{p}<0.01$.

tion of the surface immunophenotype of AML-LSCs we found that AML show evidence of a hierarchical cellular organization (Fig. 1). We also recognized that the composition of LSC phenotypes is associated with AML phenotypes. For example, secondary AML patients showed higher fraction of MPP-like LSCs compared to de novo AML patients (Fig. 2). Given that MPP-like LSCs are at the apex of LSC hierarchy, these cells are thought capable of initiating AML [4] and able to protect themselves from chemotherapy via ATP-binding cassette transporters [31-33]. In this regard, the higher expression of MPPlike LSCs could explain the poor response to standard treatments traditionally associated with secondary AML. Furthermore, the higher expression of MPP-like LSCs in post-MPN AML compared to post-MDS AML could explain the dismal prognosis associated with post-MPN AML, despite the relative indolent clinical course in their chronic phase and the presence of druggable target.

Interestingly, age did not seem to affect the composition of LSCs (Supplementary Fig. 1). The expression of $\mathrm{CD}_{34}{ }^{+} \mathrm{CD}_{3} 8^{-}$cell fraction was higher in younger patients, but this was probably due to the higher total number of cells in younger patients. When each LSC phenotype was considered, there was no difference between age groups. From clinical point of view, this finding implies two things: (1) this could explain the unsatisfactory RFS with hypomethylating agents (HMA) alone, and (2) this supports the role of allogeneic stem cell transplantation in elderly AML patients and at the same time highlights the unmet medical needs for better transplant techniques in such population. Whilst it is true that HMA has broadened the therapeutic options for elderly AML, since LSCs are reported to be more hypomethylated 


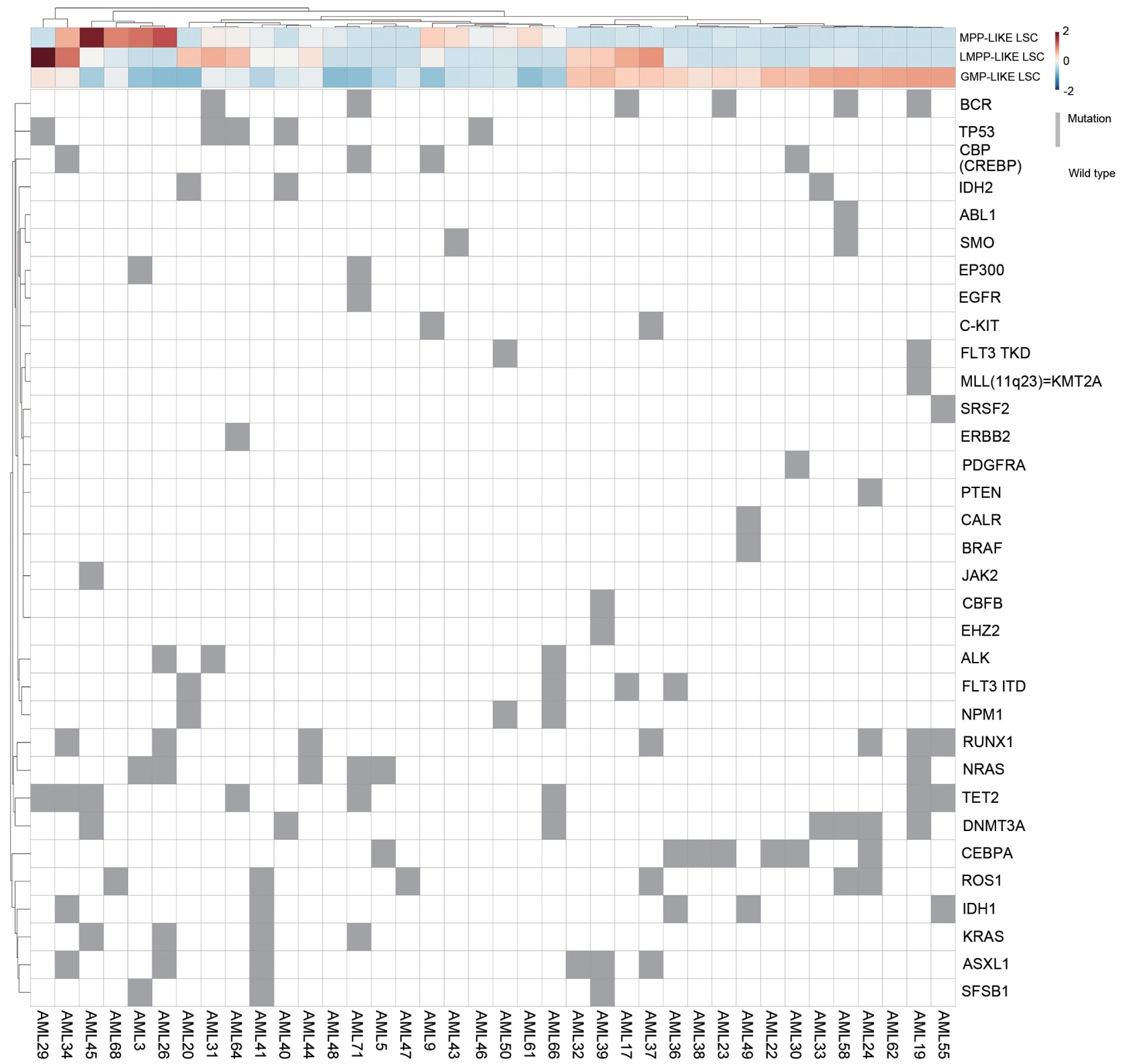

Figure 4. The dominant leukemic stem cell (LSC) phenotype and heatmap of mutations. MPP, multipotent progenitor; LMPP, lymphoid primed multipotent progenitor; GMP, granulocyte-macrophage progenitor; AML, acute myeloid leukemia.

compared to leukemic blasts to begin with, these agents cannot eradicate LSCs hence the short RFS [34]. Thus, allogeneic stem cell transplantation remains the only currently available option of eradicating LSCs [6].

Moving onto the association between LSCs, gene mutations and prognostic implications, we found that LSC phenotypes by themselves do not predict survival out- comes but when taken in together with gene mutations they carry certain predictive values. It is true that AML risk stratification has been refined considerably with the advent of NGS. However, even within the same risk group some patients do much worse than others. KRAS/ NRAS mutations deserve special attention in this regard. It has been found that there is a sequential order for the 
MPP-like LSC
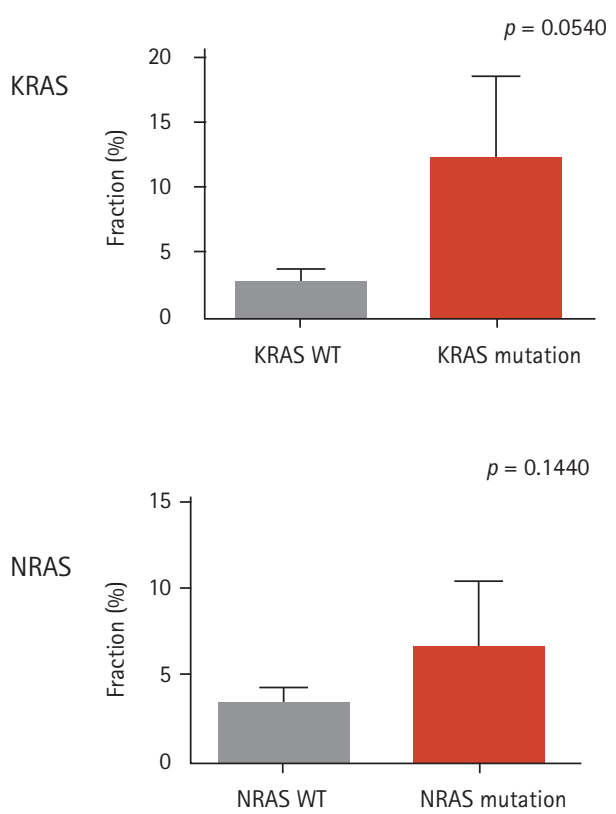

TP53

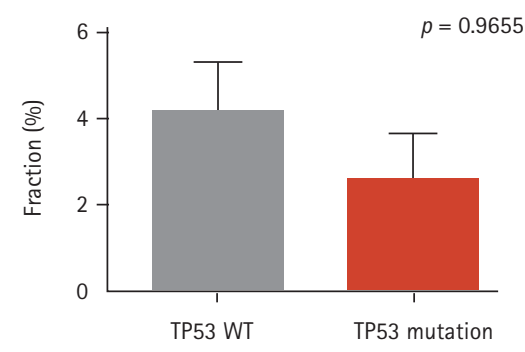

ASXL1

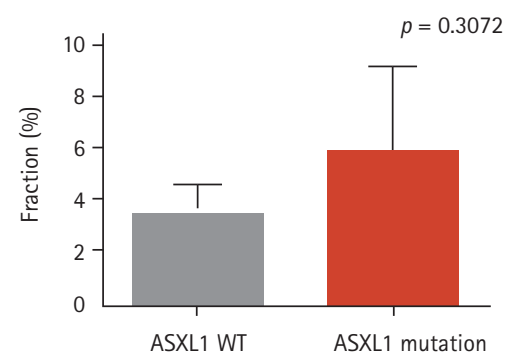

LMPP-like LSC
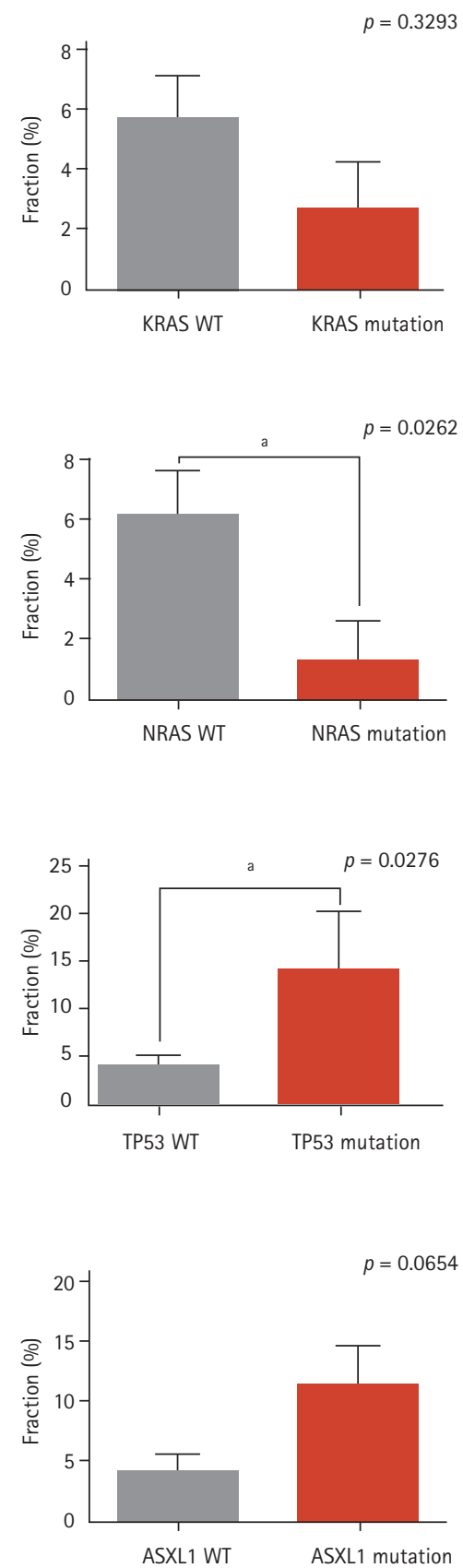

GMP-like LSC
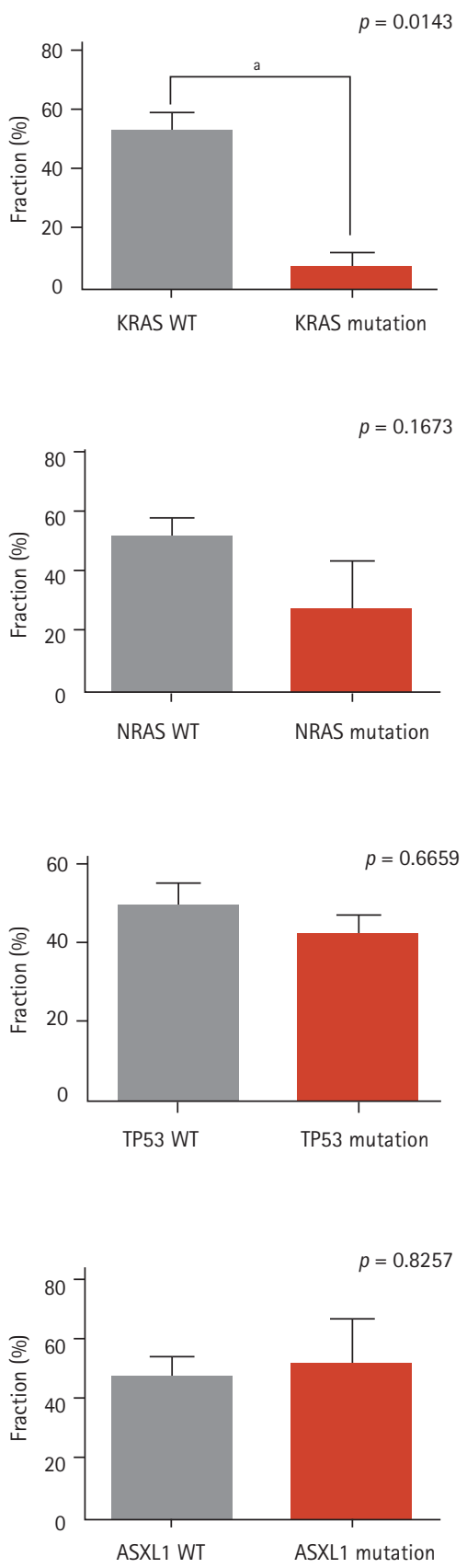

Figure 5. The relationship between individual gene mutation and leukemic stem cells (LSCs) phenotypes. Error bars indicate mean \pm standard deviations. MPP, multipotent progenitor; LMPP, lymphoid primed multipotent progenitor; GMP, granulocyte-macrophage progenitor; WT, wild type. ${ }^{\mathrm{a}} \mathrm{p}<0.05$.

acquisition of AML defining gene mutations during leukemogenesis. Somatic mutations in signaling pathways that drive proliferation, such as KRAS/NRAS, are considered later events in AML transformation [35,36].
In our samples the variant allele frequencies of KRAS/ NRAS mutations were less than $10 \%$ in most patients, confirming these mutations are acquired later during clinical course. Whether RAS mutations confer prog- 
nostic values or not is yet to be settled [37-39], thus they are not incorporated in the current risk stratification schema [14]. Although the relationship is somewhat tenuous, we showed that RAS mutants might actually be associated with worse prognosis because they tend to carry higher fraction of MPP-like LSCs (Fig. 5).

The obvious limitation of our study includes the small number of patients included. Another pitfall of our study is that the NGS was carried out with DNA extracted from bone marrow samples at diagnosis, rather than with the gated LSCs. However, we believe the value of our study lies in that it contains adequate scientific clues to inspire future studies regarding the genetics of LSC and their prognostic values. Given that merging of data on genomic and epigenomic landscape of AML with biology of LSCs is still in its infancy, our data might provide certain clues for further investigations.

In conclusion, our findings provide better insights into the characteristics and clinical implications of LSC. Although in a small scale, we provide evidence that specific LSC phenotypes are associated with certain mutations thus should be in the AML risk stratification process.

\section{KEY MESSAGE}

1. Leukemic stem cells (LSCs) are associated with clinical outcomes in acute myeloid leukemia (AML) patients, supporting the significance of cancer stem cell model for human AML.

2. The composition of LSC phenotypes is associated with AML phenotypes. For example, secondary AML patients showed higher fraction of multipotent progenitor-like LSCs compared to de novo AML patients.

3. LSC phenotypes, when taken in together with gene mutations, carry certain predictive values. This can explain why even within the same risk group some patients do much worse than others.

\section{Conflict of interest}

No potential conflict of interest relevant to this article was reported.

\section{Acknowledgments}

This work was supported by the National Research Foundation of Korea(NRF) Grant(No.NRF-2016R1A5A1011974).

The abstract of this manuscript has been presented as a poster at the 61st American Society of Hematology Annual Meeting, 2019, Orlando, FL, USA.

\section{REFERENCES}

1. Cancer Genome Atlas Research Network, Ley TJ, Miller $\mathrm{C}$, et al. Genomic and epigenomic landscapes of adult de novo acute myeloid leukemia. N Engl J Med 2013;368:2059-2074.

2. Papaemmanuil E, Gerstung M, Bullinger L, et al. Genomic classification and prognosis in acute myeloid leukemia. N Engl J Med 2016;374:2209-2221.

3. Estey EH. Acute myeloid leukemia: 2019 update on risk-stratification and management. Am J Hematol 2018;93:1267-1291.

4. Thomas D, Majeti R. Biology and relevance of human acute myeloid leukemia stem cells. Blood 2017;129:15771585 .

5. Pollyea DA, Gutman JA, Gore L, Smith CA, Jordan CT. Targeting acute myeloid leukemia stem cells: a review and principles for the development of clinical trials. Haematologica 2014;99:1277-1284.

6. Pollyea DA, Jordan CT. Therapeutic targeting of acute myeloid leukemia stem cells. Blood 2017;129:1627-1635.

7. Riether C, Schurch CM, Ochsenbein AF. Regulation of hematopoietic and leukemic stem cells by the immune system. Cell Death Differ 2015;22:187-198.

8. Taussig DC, Miraki-Moud F, Anjos-Afonso F, et al. Anti-CD38 antibody-mediated clearance of human repopulating cells masks the heterogeneity of leukemia-initiating cells. Blood 2008;112:568-575.

9. Plesa A, Dumontet C, Mattei E, et al. High frequency of $\mathrm{CD}_{34+C D_{3} 8-/ l o w}$ immature leukemia cells is correlated with unfavorable prognosis in acute myeloid leukemia. World J Stem Cells 2017;9:227-234.

10. Kreso A, Dick JE. Evolution of the cancer stem cell model. Cell Stem Cell 2014;14:275-291.

11. Vardiman JW, Thiele J, Arber DA, et al. The 2008 revision of the World Health Organization (WHO) classification of myeloid neoplasms and acute leukemia: rationale and important changes. Blood 2009;114:937-951. 
12. Brothman AR, Persons DL, Shaffer LG. Nomenclature evolution: changes in the ISCN from the 2005 to the 2009 edition. Cytogenet Genome Res 2009;127:1-4.

13. Slovak ML, Kopecky KJ, Cassileth PA, et al. Karyotypic analysis predicts outcome of preremission and postremission therapy in adult acute myeloid leukemia: a Southwest Oncology Group/Eastern Cooperative Oncology Group Study. Blood 2000;96:4075-4083.

14. Dohner H, Estey E, Grimwade D, et al. Diagnosis and management of AML in adults: 2017 ELN recommendations from an international expert panel. Blood 2017;129:424-447.

15. Goardon N, Marchi E, Atzberger A, et al. Coexistence of LMPP-like and GMP-like leukemia stem cells in acute myeloid leukemia. Cancer Cell 2011;19:138-152.

16. Vergez F, Green AS, Tamburini J, et al. High levels of $\mathrm{CD}_{34}+\mathrm{CD}_{3} 81$ ow/-CD123+ blasts are predictive of an adverse outcome in acute myeloid leukemia: a Groupe Ouest-Est des Leucemies Aigues et Maladies du Sang (GOELAMS) study. Haematologica 2011;96:1792-1798.

17. Li H. Exploring single-sample SNP and INDEL calling with whole-genome de novo assembly. Bioinformatics 2012;28:1838-1844.

18. Van der Auwera GA, Carneiro MO, Hartl C, et al. From FastQ data to high confidence variant calls: the Genome Analysis Toolkit best practices pipeline. Curr Protoc Bioinformatics 2013;43:11.10.1-11.10.33.

19. Wei Z, Wang W, Hu P, Lyon GJ, Hakonarson H. SNVer: a statistical tool for variant calling in analysis of pooled or individual next-generation sequencing data. Nucleic Acids Res 2011;39:e132.

20. Wilm A, Aw PP, Bertrand D, et al. LoFreq: a sequence-quality aware, ultra-sensitive variant caller for uncovering cell-population heterogeneity from high-throughput sequencing datasets. Nucleic Acids Res 2012;40:11189-201.

21. Chen X, Schulz-Trieglaff O, Shaw R, et al. Manta: rapid detection of structural variants and indels for germline and cancer sequencing applications. Bioinformatics 2016;32:1220-1222.

22. Rausch T, Zichner T, Schlattl A, Stutz AM, Benes V, Korbel JO. DELLY: structural variant discovery by integrated paired-end and split-read analysis. Bioinformatics 2012;28:i333-i339.

23. Oesper L, Satas G, Raphael BJ. Quantifying tumor heterogeneity in whole-genome and whole-exome sequencing data. Bioinformatics 2014;30:3532-3540.
24. Talevich E, Shain AH, Botton T, Bastian BC. CNVkit: genome-wide copy number detection and visualization from targeted DNA sequencing. PLoS Comput Biol 2016;12:e1004873.

25. Cingolani P, Platts A, Wang le L, et al. A program for annotating and predicting the effects of single nucleotide polymorphisms, SnpEff: SNPs in the genome of Drosophila melanogaster strain w1118; iso-2; iso-3. Fly (Austin) 2012;6:80-92.

26. O'Leary NA, Wright MW, Brister JR, et al. Reference sequence (RefSeq) database at NCBI: current status, taxonomic expansion, and functional annotation. Nucleic Acids Res 2016;44(D1):D733-D745.

27. Tate JG, Bamford S, Jubb HC, et al. COSMIC: the Catalogue Of Somatic Mutations In Cancer. Nucleic Acids Res 2019;47(D1):D941-D947.

28. Sherry ST, Ward MH, Kholodov M, et al. dbSNP: the NCBI database of genetic variation. Nucleic Acids Res 2001;29:308-311.

29. Landrum MJ, Lee JM, Riley GR, et al. ClinVar: public archive of relationships among sequence variation and human phenotype. Nucleic Acids Res 2014;42(Database issue):D980-D985.

30. Lek M, Karczewski KJ, Minikel EV, et al. Analysis of protein-coding genetic variation in 60,706 humans. Nature 2016;536:285-291.

31. Dean M, Fojo T, Bates S. Tumour stem cells and drug resistance. Nat Rev Cancer 2005;5:275-284.

32. Raaijmakers MH. ATP-binding-cassette transporters in hematopoietic stem cells and their utility as therapeutical targets in acute and chronic myeloid leukemia. Leukemia 2007;21:2094-2102.

33. Wang X, Huang S, Chen JL. Understanding of leukemic stem cells and their clinical implications. Mol Cancer 2017;16:2.

34. Craddock C, Quek L, Goardon N, et al. Azacitidine fails to eradicate leukemic stem/progenitor cell populations in patients with acute myeloid leukemia and myelodysplasia. Leukemia 2013;27:1028-1036.

35. Corces-Zimmerman MR, Hong WJ, Weissman IL, Medeiros BC, Majeti R. Preleukemic mutations in human acute myeloid leukemia affect epigenetic regulators and persist in remission. Proc Natl Acad Sci U S A 2014;111:25482553.

36. Corces-Zimmerman MR, Majeti R. Pre-leukemic evolution of hematopoietic stem cells: the importance of early 
mutations in leukemogenesis. Leukemia 2014;28:22762282.

37. Zhou JD, Yao DM, Li XX, et al. KRAS overexpression independent of RAS mutations confers an adverse prognosis in cytogenetically normal acute myeloid leukemia. Oncotarget 2017;8:66087-66097.
38. DiNardo CD, Cortes JE. Mutations in AML: prognostic and therapeutic implications. Hematology Am Soc Hematol Educ Program 2016;2016:348-355.

39. Liu X, Ye Q, Zhao XP, et al. RAS mutations in acute myeloid leukaemia patients: a review and meta-analysis. Clin Chim Acta 2019;489:254-260. 


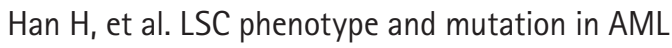

Supplementary Table 1. LSC phenotype according to AML risk stratification by cytogenetics only

\begin{tabular}{|c|c|c|c|c|}
\hline Conventional risk & Low & Intermediate & High & $p$ value \\
\hline Total no. & 6 & 38 & 8 & NA \\
\hline MPP-like LSC & $4.30 \pm 5.06$ & $2.52 \pm 4.59$ & $7 \cdot 52 \pm 8.84$ & 0.0675 \\
\hline LMPP-like LSC & $8.42 \pm 8.31$ & $3.89 \pm 5.80$ & $10.07 \pm 11.58$ & 0.0273 \\
\hline GMP-like LSC & $28.46 \pm 38.62$ & $49.17 \pm 36.30$ & $41.10 \pm 15.12$ & 0.3196 \\
\hline De novo AML no. & 6 & 32 & 2 & NA \\
\hline MPP-like LSC & $4.30 \pm 5.06$ & $1.19 \pm 1.95$ & $9.54 \pm 8.56$ & 0.0500 \\
\hline LMPP-like LSC & $8.42 \pm 8.31$ & $3.74 \pm 5.29$ & $2.04 \pm 1.54$ & 0.1748 \\
\hline GMP-like LSC & $28.46 \pm 38.62$ & $54 \cdot 30 \pm 35.15$ & $36.30 \pm 8.78$ & 0.1742 \\
\hline Secondary AML no. & 0 & 6 & 6 & NA \\
\hline MPP-like LSC & NA & $9.62 \pm 7.80$ & $6.85 \pm 9.62$ & 0.4848 \\
\hline LMPP-like LSC & NA & $4.68 \pm 8.67$ & $12.75 \pm 12.36$ & 0.0921 \\
\hline GMP-like LSC & NA & $21.78 \pm 31.90$ & $42.70 \pm 17.10$ & 0.2403 \\
\hline
\end{tabular}

Values are presented as mean $\pm \mathrm{SD}$.

LSC, leukemic stem cell; AML, acute myeloid leukemia; NA, not applicable; MPP, multipotent progenitor; LMPP, lymphoid primed multipotent progenitor; GMP, granulocyte-monocyte progenitor. 
Supplementary Table 2. LSC phenotype according to AML risk stratification based on NGS data

\begin{tabular}{|c|c|c|c|c|}
\hline Combined risk ${ }^{\mathrm{a}}$ & Low & Intermediate & High & $p$ value \\
\hline Total no. & 7 & 19 & 12 & NA \\
\hline MPP-like LSC & $2.58 \pm 3.46$ & $3.32 \pm 6.90$ & $5.55 \pm 6.57$ & 0.2699 \\
\hline LMPP-like LSC & $1.54 \pm 2.23$ & $2.95 \pm 4.72$ & $11.47 \pm 10.20$ & 0.0052 \\
\hline GMP-like LSC & $44.46 \pm 34.23$ & $52.18 \pm 39.18$ & $43.59 \pm 23.24$ & 0.7741 \\
\hline De novo AML no. & 7 & 15 & 6 & NA \\
\hline MPP-like LSC & $2.58 \pm 3.46$ & $0.73 \pm 1.69$ & $3.74 \pm 5.99$ & 0.2739 \\
\hline LMPP-like LSC & $1.54 \pm 2.23$ & $3.37 \pm 5.14$ & $7.51 \pm 6.36$ & 0.0401 \\
\hline GMP-like LSC & $44.46 \pm 34.23$ & $64.89 \pm 33.84$ & $47.31 \pm 27.00$ & 0.2731 \\
\hline Secondary AML no. & 0 & 4 & 6 & NA \\
\hline MPP-like LSC & NA & $13.04 \pm 10.61$ & $7.36 \pm 7.15$ & 0.4762 \\
\hline LMPP-like LSC & NA & $1.37 \pm 2.49$ & $15.44 \pm 12.29$ & 0.0871 \\
\hline GMP-like LSC & NA & $4.50 \pm 5.10$ & $39.87 \pm 20.63$ & 0.0667 \\
\hline
\end{tabular}

Values are presented as mean $\pm \mathrm{SD}$.

LSC, leukemic stem cell; AML, acute myeloid leukemia; NGS, next generation sequencing; NA, not applicable; MPP, multipotent progenitor; LMPP, lymphoid primed multipotent progenitor; GMP, granulocyte-monocyte progenitor.

${ }^{a}$ Combined risk refers to risk stratification based on available next generation sequencing data including TP53, ASXL1, RUNX1 mutation status. 
Han $\mathrm{H}$, et al. LSC phenotype and mutation in AML

Supplementary Table 3. Treatment schema and outcomes

\begin{tabular}{|c|c|c|c|}
\hline Variable & Total & De novo AML & Secondary AML \\
\hline \multicolumn{4}{|l|}{ Initial treatment } \\
\hline No treatment & $3(5.8)$ & $1(2.5)$ & $2(16.7)$ \\
\hline Low intensity treatment ${ }^{\mathrm{a}}$ & $18(34.6)$ & $14(35 \cdot 0)$ & $4(33 \cdot 3)$ \\
\hline Intensive induction ${ }^{\mathrm{a}}$ & $31(59.6)$ & $25(62.5)$ & $6(50.0)$ \\
\hline CR achievement with induction ${ }^{b}$ & $23(74.2)$ & $20(80.0)$ & $3(50.0)$ \\
\hline Induction TRM & $4(12.9)$ & $4(16.0)$ & o \\
\hline Upfront HSCT & $13(25.0)$ & $8(20.0)$ & $5(41.7)$ \\
\hline \multicolumn{4}{|l|}{ HSCT donor } \\
\hline Matched related & $5(38.5)$ & $5(62.5)$ & $\mathrm{O}$ \\
\hline Matched unrelated & $4(30.8)$ & $3(37 \cdot 5)$ & $1(20.0)$ \\
\hline Others $^{c}$ & $4(30.8)$ & o & $4(80.0)$ \\
\hline \multicolumn{4}{|l|}{ HSCT conditioning } \\
\hline Myeloablative & $7(53.8)$ & $3(37 \cdot 5)$ & $4(80.0)$ \\
\hline Reduced intensity & $6(46.2)$ & $5(62.5)$ & $1(20.0)$ \\
\hline Relapse & $29(55.8)$ & $23(57 \cdot 5)$ & $6(50.0)$ \\
\hline Relapse free survival, mon & $10.8 \pm 8.7$ & $11.3 \pm 8.8$ & $9.0 \pm 8.6$ \\
\hline Death & $18(34 \cdot 6)$ & $14(35 \cdot 0)$ & $4(33 \cdot 3)$ \\
\hline Overall survival, mon & $12.3 \pm 8.8$ & $13.2 \pm 8.8$ & $9.3 \pm 8.4$ \\
\hline
\end{tabular}

Values are presented as number (\%) or mean \pm SD.

AML, acute myeloid leukemia; CR, complete remission; TRM, treatment related mortality; HSCT, hematopoietic stem cell transplantation; NA, not applicable.

${ }^{a}$ Low intensity treatment refers to hypomethylating agents, venetoclax, low dose cytarabine; while intensive induction refers to standard $3+7$ induction chemotherapy.

${ }^{\mathrm{b}}$ For patients undergoing standard induction chemotherapy.

${ }^{\mathrm{c}}$ Others include mismatched related donors and cord blood. 

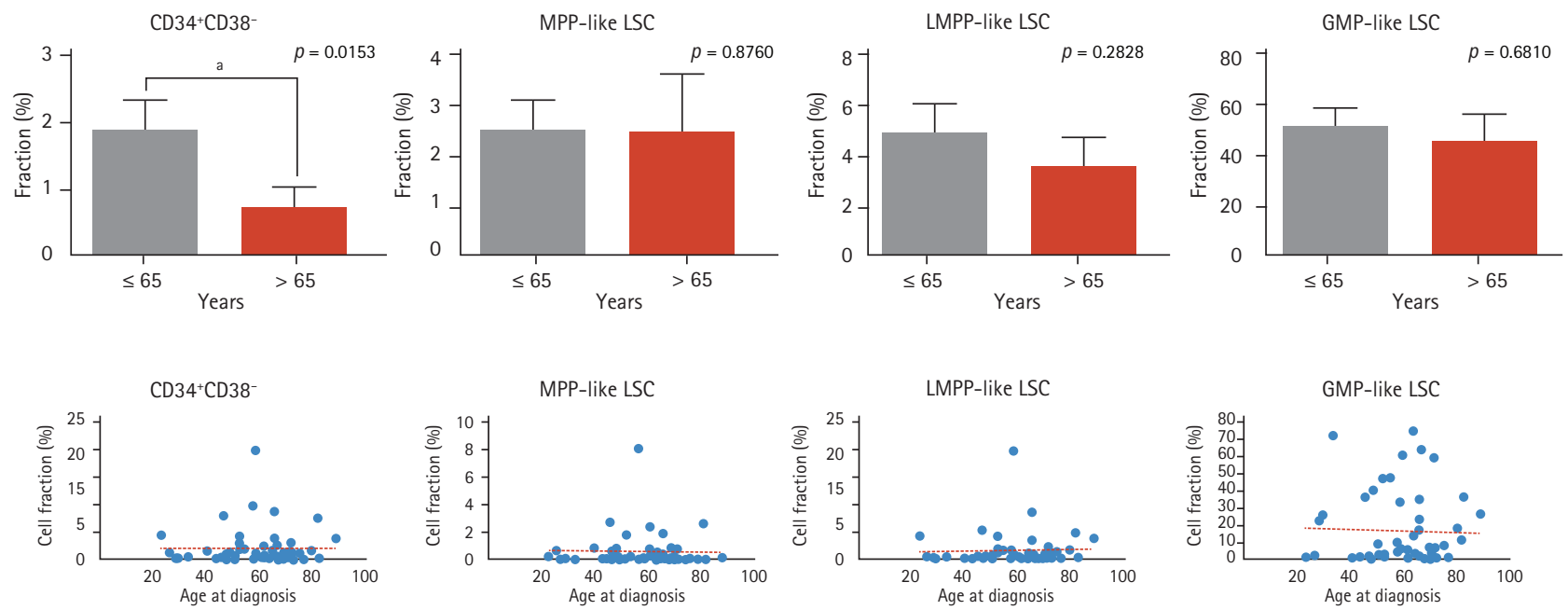

Supplementary Figure 1. The age at acute myeloid leukemia diagnosis and leukemic stem cell (LSC) phenotypes. MPP, multipotent progenitor; LMPP, lymphoid primed multipotent progenitor; GMP, granulocyte-monocyte progenitor. 
A
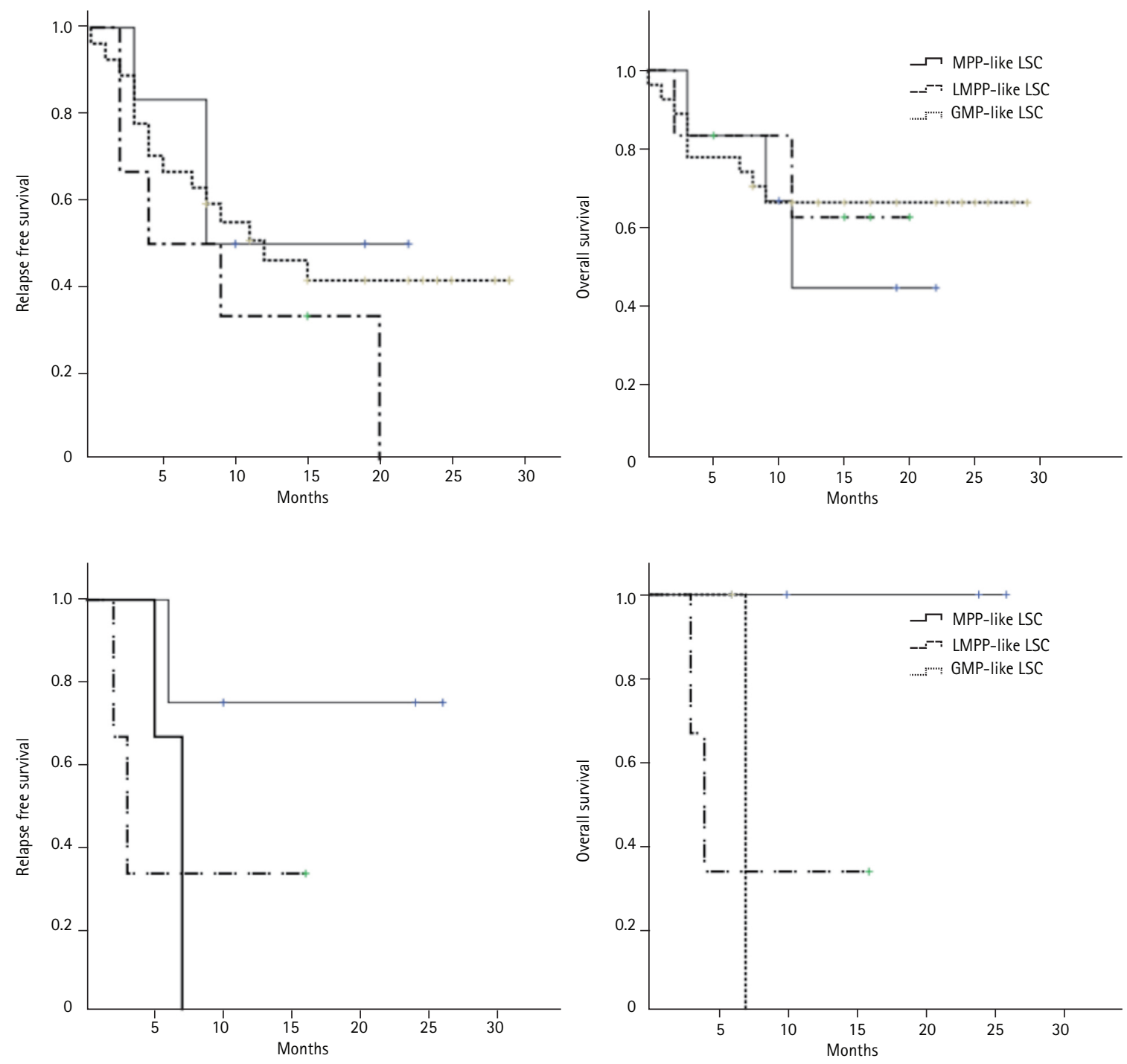

B

Supplementary Figure 2. Survival analyses. (A) Relapse free survival (RFS) and overall survival (OS) of 39 de novo acute myeloid leukemia (AML) patients undergoing treatment. (B) RFS and OS of 10 secondary AML patients undergoing treatment. MPP, multipotent progenitor; LSC, leukemic stem cell; LMPP, lymphoid primed multipotent progenitor; GMP, granulocyte-monocyte progenitor. 